\title{
HYDROGEN GAS EMBRITTLEMENT
}

\author{
by
}

H. H. Johnson

Invited Paper Presented at the Conference on

"Hydrogen in Metals: Effect on Properties, Selection and Design", held at Seven Springs,

Pennsylvania in September 1973

This report NOTICE

sponsored was prepared as an account of work the United Sthe United States Government. Neither Commission thes nor the United States Atomic Energy heir contractor any of their employees, nor any of makes any warranty, legal liability or responsibility for the or assumes any pleteness or usefulness of any inform accuracy, comproduct or process disclosed, would not infringe privately owned rights.

$$
\text { L }
$$

\author{
November 1973 \\ Cornell University \\ Ithaca, New York 14850 \\ November 1973
}

Report \#2121

Is sued by

The Materials Science Center

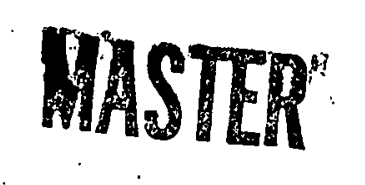

DISTRIBUTION OF THIS DOCUMENT IS UNLIMITEO 


\section{DISCLAIMER}

This report was prepared as an account of work sponsored by an agency of the United States Government. Neither the United States Government nor any agency Thereof, nor any of their employees, makes any warranty, express or implied, or assumes any legal liability or responsibility for the accuracy, completeness, or usefulness of any information, apparatus, product, or process disclosed, or represents that its use would not infringe privately owned rights. Reference herein to any specific commercial product, process, or service by trade name, trademark, manufacturer, or otherwise does not necessarily constitute or imply its endorsement, recommendation, or favoring by the United States Government or any agency thereof. The views and opinions of authors expressed herein do not necessarily state or reflect those of the United States Government or any agency thereof. 


\section{DISCLAIMER}

Portions of this document may be illegible in electronic image products. Images are produced from the best available original document. 


\author{
HYDROGEN GAS EMBRITTLEMENT \\ H. H. Johnson \\ Department of Materials Science and Engineering \\ Cornell University \\ I thaca, New York 14850
}

\begin{abstract}
The basic characteristics of the brittleness induced in many alloys by hydrogen gas are reviewed, discussed, and contrasted with the characteristics of classical hydrogen brittleness. The diffusion penetration distance of hydrogen ahead of a moving crack is estimated. The influence of elastic stresses and plastic deformation upon hydrogen distribution is qualitatively assessed.
\end{abstract}




\title{
HYDROGEN GAS EMBRITTLEMENT
}

\author{
H. H. Johnson \\ $=$ \\ Department of Materials Science and Engineering \\ Cornell University..
thaca., New York 14850 -
}

\section{INTRODUCTION}

It has been established in the past decade that external hydrogen gas causes brittleness in many alloy systems, including steels $(1-5)$, titanium and titanium alloys $(i, 6-7)$, and nickel and nickel-base alloys $(1,5$, $8-9)$. Hydrogen pressures from much less than one atmosphere $\left(10^{5} \mathrm{~N} / \mathrm{m}^{2}\right)$ to 10,000 psi $\left(6.8 \times 10^{7} \mathrm{~N} / \mathrm{m}^{2}\right)$ have been used, and it is clear that in general susceptibility to hydrogen gas increases with pressure. Parameters measured as indices of brittleness include elongation, reduction of area, notch tensile strength, time to failure, crack growth rate, threshold stress and threshold stress intensity: In common with classical hydrogen brittleness, the notch and flaw-associated properties are far more sensitive to hydrogen gas than are the unnotched properties, and brittleness is more evident in high strength alloys than low strength alloys.

The lattice hydrogen concentrations corresponding to the pressure range studied are quite small. For iron the lattice concentration in $\mathrm{cm}^{3}\left(\mathrm{H}_{2}, N T P\right) / \mathrm{cm}^{3} \mathrm{Fe}$ may be estiriated from

$$
c=3.7 p^{\frac{1}{2}} \exp (-6500 / R T),
$$

obtained from an analysis(10) of data from different studies conducted at temperatures well above $300^{\circ} \mathrm{K}$. 
Extrapolation of this equation is the only method available to estimate equilibrium solubilities at lower temperatures, since the concentrations are well below the limits of present experimental techniques.

A lattice concentration of approximately four $H$. atoms per $10^{8} \mathrm{Fe}$ atoms is in equilibrium with hydrogen gas to $300^{\circ} \mathrm{K}$ and one atmosphere pressure. At 10,000 psi the lattice concentration is increased by a factor of about 26, while at 0.1 atom it is decreased by about 0.31 . These are very small concentrations, and it is most improbable that brittleness can be associated with hydrogen dispersed on this scale. Iron has the lowest solubility for hydrogen of the metals that are normally considered in connection with hydrogen brittleness. In nickel the solubility is about 175 times that in iron under identical conditions, and for Ti, $\mathrm{T}, \mathrm{Nb}$, and $\mathrm{Zr}$ the solubility is much larger.

For steels the hydrogen concentrations commonly reported at $300^{\circ} \mathrm{K}$ are orders of magnitude larger, say one to five parts per million, than the estimated lattice solubilities. Virtually all of this hydrogen is trapped at structural imperfections such as microvoids, dislocation aggregates, matrix-inclusion interfaces, impurity atoms, etc. Trapping phenomena are equally important in other metals, but the higher lattice solubilities diminish the effect of trapping upon total solubility. Detailed characterization of trapping behavior is a prerequisite to future mechanistic studies of hydrogen brittieness, since some traps may be the source of damaging hydrogen or crack initiation sites, and others may act as innocuous sinks.

It has been reported(4) that there is little correlation between the susceptibilities of various alloys to hydrogen gas and classical hydrogen brittleness. Although there is a-reasonable correlation of susceptibilities for structural steels, high strength nickel base alloys suffer little embrittlement after electrolytic charging with hydrogen, but are extremely susceptible to 10,000 psi $\left(6.8 \times 10^{7} \mathrm{Ni} \mathrm{n}^{2}\right)$ hydrogen gas $(4)$. It has been suggested(8) that these observations may be explained by trapping of internal hydrogen at voids created at matrix-particle interfaces during deformation; the lack of brittleness is presumed to correlate with depletion of the matrix hydiogen. In hydrogen gas there is a continual supply of hydrogen at the crack tip, and brittleness is observed. This concept illustrates the potential importance of trapping in interpreting 
hydrogen brittleness.

\section{COMMENTS ON PHENOMENOLOGICAL CHARACTERISTICS}

The site of crack initiation is an important aspect of the question of whether or not there are essential differences between classical and hydrogen gas brittleness. In classical hydrogen brittleness it is well established(11) that hydrogen induced crack initiation can occur below notch tip surfaces, and that the initiation site moves closer to the surface as the notch sharpness is increased. This experimental approach has apparently not been attempted for hydrogen gas brittleness, but from observations of surface cracking(4) and crack growth kinetics(3) many investigators are clearly convinced that hydrogen gas cracks initiate at external surfaces. However, the only quantitative theory employing that assumption has been criticized $(12,13)$. If hydrogen gas cracks do initiate exclusively at external surfaces, then some differences in the characteristics of hydrogen gas and cllassical brittleness are to be expected.

The phenomenological characteristics of classical and hydrogen gas brittleness at low pressure are similar. With pre-cracked high strength steel specimens exposed to a fixed low hydrogen pressure(3), the brittleness decreased with increasing displacement rate, was a maximum in rate near $300^{\circ} \mathrm{K}$ and decreased at both lower and higher temperatures $(3,14)$; similar behavior patterns are observed with classical hydrogen brittleness(15). However, the kinetic parameters, especially the activation energy, are apparently not identical in magnitude to tiose normally associated with classical hydrogen brittleness, and the cause of this is not clear. It may reflect differences in crack initiation site. Par. tial dissociation of hydrogen gas, which increases the atomic hydrogen concentration in the gas phase, markedly increased(16) the crack growth rate in parallel to the more severe classical hydrogen brittleness observed with higher internal hydrogen concentrations(17).

Pre-exposure to hydrogen, with or without stress, does not markedly increase the eventual brittleness as measured under $r$ ising load. This rather surprising result has been observed with several steels exposed to both low $(1,2)$ and high $(1,18-19)$ pressure hydrogen. With pre-cracked specimens(2) the threshold stress intensity and crack growth rate are not influenced by prior 
exposure to low pressure hydrogen, while with high pressure hydrogen the notch strength is similarly unaffected by prior exposure $(18,19)$. Furthermore, with unnotched specimens the shallow surface cracks associated with hydrogen exposure were much more numerous near the fracture and were frequently restricted to the necked-down region.

These observations suggest(19) that a critical amount of deformation is necessary before hydrogen can interact with the surface and perhaps diffuse into the interior. It may be necessary for the deformation to rupture surface oxides and expose clean metai before the hydrogen can interact.

The occurrence of hydrogen gas brittleness is markedly sensitive to gas purity, an effect which does not have a direct analogue in classical hydrogen brittleness. For quenched and tempered steels at strength in excess of $200 \mathrm{KSI}$ and under monotonic load, the addition of as little as $0.4 \%$ oxygen to hydrogen will eliminate hydrogen-induced crack growth $(2,74)$. At lower oxygen concentrations the threshold stress intensity is approximately linear with oxygen, a result which is only slightly influenced by temperature over the range from 2 to $78^{\circ} \mathrm{C}(14)$. With cyclic loading the picture is more complex; with strength levels in excess of $200 \mathrm{KSI}$ oxygen is partially or completely ineffective in stopping hydrogen-induced crack growth $(14,15)$, but at lower strength levels oxygen additions do eliminate hydrogenacceleriated fatigue crack growth(14).

The straightforward interpretation is that oxygen adsorbs preferentially on the surface, thereby excluding hydrogen from contact with the crack tip region. This suggests that brittleness is not controlled simply by the strength of interaction between a surface atom and an adsorbed species(20), for oxygen is more tightly bound, and yet oxygen does not cause crack growth in high strength steels. This particular observation is more compatible with hydrogen diffusion into the steel and sub-surface crack initiation, since hydrogen has $\bar{c}$. much higher diffusivity than does oxygen.

\section{HYDROGEN NEAR MOVING CRACKS}

An estimate of the hydrogen diffusion distance ahead of a moving crack would be useful in discussions of mechanism. With hydrogen gas brittleness the high 
diffusivity, orders of magnitude larger than for any other solute, may allow hydrogen to penetrate a significant distance ahead of the moving crack tip. An estimate of the diffusion distance must consider the crack to be moving; from dimensional considerations the penetration distance should depend on the parameter $D / V$, where $D$ is the diffusivity and $V$ the crack velocity. The relation between the concentration profile and the parameter D/V will depend upon the crack geometry and diffusion field boundary conditions.

The following colculations assume steady state crack growth, which may be defined as a constant crack velocity $V$. The hydrogen concentration profile is then time independent in the reference frame moving at $V$, and this simplifies the analysis. Constant crack velocities are experimentally realizable through fracture mechanics techniques.

An exact model of the moving crack-hydrogen diffusion system cannot be constructed at present. However, two limiting cases may be treated, which should encompass the range of possibilities. In the first model it is assumed that hydrogen enters the steel only at the tip of the moving crack, and that the crack sides are impermeable. This is physically realistic in view of the previous observations that crack growth is not accelerated by exposure to hydrogen before stress application. Evidently hydrogen enters only through the freshly strained metal at the crack tip, which is presumably very clean, and the crack walls are passive, perhaps because of oxygen contamination.

The moving crack can then be assumed to act as a moving line source of hydrogen; the corresponding diffusion field follows directiy from the analyses of 1) heat flow from a moving line source(21), or 2) the point defect field surrounding a moving dislocation!??). In polar coordinates $(r, \theta)$ centered upon the moving line source, the concentration profile may be expressed as

$$
c(r, \theta)=\beta K_{0}\left(\frac{V r}{20}\right) \exp \left(-\frac{V r \cos \theta}{20}\right)
$$

$K_{0}=$ modified Bessel function of second kind of order 2ero. Since this expression is singular at $r=0$, the constant $\beta$ must be determined by setting a radius $r_{0}$ to the singularity, with a corresponding concentra- 
tion $c_{0}$ at $r_{0}$. $r_{0}$ would be similar in magnitude to the crack tip radius. The concentration profile in reduced variables is then

$$
\frac{c}{c_{0}}=\frac{k_{0}\left(\frac{V r}{2 D}\right) \exp \left(-\frac{V r \cos \theta}{2 D}\right)}{k_{0}\left(\frac{V r_{0}}{2 D}\right) \exp \left(-\frac{V r_{0} \cos \theta}{20}\right)},
$$

which allows an assessment of crack tip radius upon the extent of the moving diffusion field.

The second model considers the other extreme, that hydrogen enters from every segment of the crack surface, and that the moving crack is both very thin and semjinfinite in length. This assumes the crack walls to be completely permeable, which may be reasonable for crack propagation in aqueous media. With $c_{0}$ as the concentration at the crack surface, the concentration profile in reduced variables may be..taken from a recent analysis (23) of dendrite and Widmanstätten plate growth as

$$
\begin{aligned}
& c / c_{0}=\operatorname{erfc}\left\{\left(\frac{V}{2 D}\right)^{\frac{1}{2}}(x+r)^{\frac{1}{2}}\right\} \\
& x=\text { distance ahead of noving crack } \\
& r=\left(x^{2}+y^{2}\right)^{\frac{1}{2}}
\end{aligned}
$$

In both models the concentration $c_{0}$ is controlled by the reaction at the crack surface between the hydrogen gas and the dissolved hydrogen. If local equili-brium prevails at the interface, then $c_{0}$ is given by the numerical estimates in an earlier section. The local equilibrium assumption is consistent with the concept of a clean and strained metal surface at the crack tip. The alternative is interface inhibition or control, which would decrease the penetration distance. In the absence of further information, it is reasonable to take the concentration at the surface as a constant independent of position on the surface.

The extent of penetration is illustrated by the calculated concentration profiles directly ahead of and 
in the plane of the moving crack, Figures 1 and 2 . The diffusivities and crack velocities used in the calculations were selected to span the range of values reported in the literature. Crack veiocities from $10^{-4}$ to $10^{-1}$ $\mathrm{cm} / \mathrm{s}$ are commonly reported for high strength steels, while for a titanium alloy crack growth rates from approximately $10^{-5}$ to $10^{-3} \mathrm{~cm} / \mathrm{s}$ have been reported $(7)$.

The $300^{\circ} \mathrm{K}$ effective hydrogen diffusivity in iron base materials is a poorly characterized parameter, with reported values varying from $10^{-9} \mathrm{~cm}^{2} / \mathrm{s}$ to $10^{-5} \mathrm{~cm}^{2} / \mathrm{s}$. This spread reflects structural trapping of hydrogen, and for that reason the lower $D$ values are gellerally associated with the higher strength and microstructurally more complex steels. However, the material ahead of a moving crack is plastically straining as lihe hydrogen moves through, and recent observations :iuggest that hydrogen transport is accelerated by simultaneous plastic deformation.. Fidelle(24) reported a sharp increase of outgassing rate when a hydrogen pressurized sphere was plastically strained, and Louthan et al. (25) observed by tritium autoradiography in a hetergeneously strained specimen that penetration is greater in the plastically strained region than in the elastic region. Plastic deformation ahead of a moving crack may therefore increase hydrogen transport; in a permeation experiment, this could be described as an increase in effective diffusivity.

It is evident from Figures 1 and 2 that a significant peretration of hydrogen ahead of the moving crack can be anticipated. Depending on the particular model and $D / V$ ratio, penetration distances from approximately $10^{-6}$ to $10^{-3} \mathrm{~cm}$ are feasible for a reduced concentration of 0.5 .

When the line and semi-infinite source models are compared, the diffusion penetration distance is of course greater for the semi-infinite source model. This is most evident at larger $D / V$ values. For smaller $D / V$ values, the concentration profiles are closer together, since under these circumstances most of the hydrogen in advance of the crack is coming from the near tip regiun. For the same reason, at the lower values of reduced concentration, the penetration distances tend to become model independent.

There are surely uncertainties in the assumptions underlying these calculations. In addition to the trapping and dynamic plasticity effects mentioned previously, 
the distribution of hydrogen in the crack tip region will be influenced by the accompanying heterogeneous stress field. This is a thermodynamic effect, with its origin in the reversible dilatation of the material under elastic stress and the positive volume change that accompanies insertion of a hydrogen interstitial in the lattice. To maintain a position independent chemical potential, hydrogen tends to concentrate in regions of positive strain and compressively strained regions are depleted. The chemical potential of the dissolved hydrogen may be written(26) as

$$
\begin{aligned}
\mu=\stackrel{0}{\mu}+\operatorname{kTen} C_{H}-P \bar{V}_{H} & (4) \\
C_{H}= & \text { hydrogen concentration } \\
P= & \text { hydrostatic component of stress field, a func- } \\
& \text { tion of position in flawed or notched specimens } \\
\bar{V}_{H}= & \begin{array}{l}
\text { phenomenological partial atomic volume of } \\
\text { hydrogen }
\end{array}
\end{aligned}
$$

The re-distribution of hydrogen then follows as

$$
c_{H}=c_{0} \exp \left(\frac{P \bar{V}_{H}}{k T}\right)
$$

$c_{0}=$ uniform concentration in absence of stress.

An upper limit to the re-distribution may be obtained if $P$ is estimated from a fracture criterion. If it is postulated that fracture initiates when the tensile stress in a local region attains the theoretical fraciare strength, which may be estimated as $E / \pi$, then the corresponding value of $P$ would be $E / 3 \pi$. This leads to a concentration enhancement of

$$
c_{H} / c_{0} \simeq 4 \times 10^{7}
$$

for iron. This number is very approximate, since because of the exponential form of equation. 5 the result is very sensitive to the fracture strength criterion 
assumed. The present choice is probably close to an upper limit.

The re-distribution effected by a stress equal to the theoretical fracture stress is indeed enormous, so. large that it might be questioned if the assumptions underlying the development of the thermodynamic equation are in anyway violated. Yet the lattice concentration corresponding to $\mathrm{C}_{\mathrm{H}} \simeq 4 \times 10^{7} \mathrm{c}_{0}$ is still not particularly large if $c_{0}$ is for an equilibrium with hydrogen gas at $1 \mathrm{~atm}$. $C_{H}$ at the theoretical fracture stren:th is then just about $2 \mathrm{H}$ atoms per Fe atom. Thus, the absolute concentration produced by a stress equal to tie theoretical fracture strength is not exceptional, a ithough the relative change is very large.

The redistribution of hydrogen in a heterugeneous stress field is therefore controlled by the position dependent triaxiality. It is a result of isotropic elasticity that the triaxiality is a harmonic function, and therefore obeys Leplace's equation,

$$
\nabla^{2} P=0
$$

at all points in the elastically strained body. It is a well known property of harmonic functions that they take on extreme values only on the boundary of the region in which they are defined, and cannot exhibit either maxima or minima except on the surface. In a specimen subject to purely elastic strain, therefore, the maximum hydrogen concentration from strain induced redistribution should be on the surface. For a notched or pre-cracked specimen, this would be at the notch or crack tip.

This picture is undoubtedly too idea? for cracks propagating in real materials, since some plastic deformation aiways precedes the growing crack. If the system is considered to be elastic-plastic, with a sharply diefined plastic enclave around the moving crack, then the considerations just discussed indicate that insofar as the elastic region is concerned, the maximum hydrogen concentration will occur on the elastic-plastic boundary.

Within the plastic region understanding of the detailed stress state is less secure. However, a recent analysis(27) which incorporates the large crack tip geometry changes in plane strain fracture indicates that 
the position of maximum plastic triaxiality is not at the crack tip surface, but rather at some distance into the plastic enclave. The distance $\delta_{t}$ to the point of maximum triaxiality was estimated as

$$
\delta_{t}=\frac{K^{2}}{2 E \sigma_{y}}
$$

$$
\begin{aligned}
K & =\text { stress intensity } \\
E & =\text { elastic modulus } \\
\sigma_{y} & =\text { yield strength }
\end{aligned}
$$

Insertion of typical numbers for high strength steels leads to $\delta_{t}$ values in the range of $10^{-5}-10^{-6} \mathrm{~cm}$, which is well within the concentration profiles reported in Figures 1 and 2 . It may be concluded thai hydrogen diffusion to the point of maximum plastic triaxiality ahead of the moving crack is a distinct possibility.

It does not necessarily follow that hydrogen will segregate to the position of maximum triaxiality in a plastic stress field. Incompressible behavior is normally assumed in plastic analysis; since volume changes under stress are the root cause of hydrogen segregation, this process may well be minimal or absent in a continuum plasticity approximation. The maximum triaxiality position would still be the site of crack initiation.

\section{COMMENTS ON MECHANISM}

The occurrence of crack growth in low pressure hydrogen is of course not compatible with a pressure mechanism of brittleness. This is self evident if the crack initiating site is on the surface. If hydrogen diffusion ahead of the crack is essential, then some of this riydrogen will surely be trapped, perhaps at microvoids created at inclusion interfaces or in grain boundaries by the local heterogeneous stress field. Although the local lattice concentration may be many orders of magnitude greater than the remote concentration, as a result of stress-induced redistribution, the amount which precipitates into microvoids should not, on thermodynamic grounds, lead to a trap hydrogen pressure any larger than the external pressure. The precipitated hydrogen could 
conceivably stabilize the deformation-induced trap by lowering its surface energy, but for the pressure to rise above the external value would require that the system move away from equilibrium, and this must be regarded as improbable.

With respect to the brittleness mechanism, it is most probable that hydrogen reduces the material cohesion at the crack initiation site by a process whose physics is as yet unclear. There is an interesting analogy with the hydrogen promoted evaporation of metal atoms from filament lips under very high electric fields, an effect which is ussfully employed in field ion microscopy. In the absence of hydrogen an electric field which is sufficient to pull atoms off the surface also generates stresses in lihe filament tip of the order of magnitude of estimated theoretical fracture stresses. These stresses are sustained without deformation because of the relative absence of defects in small filaments. With the addition of hydrogen at low partial pressures, the electric field necessary to evaporate atoms is drastically reduced for many metals, which may be viewed as a corresponding reduction in theoretical fracture strength. It has been suggested that there is an interaction between the hydrogen and the slightly exposed ion cores at the filament tip surface, which reduces the bindirg between the surface atoms and the bulk.

The analogy suggests that brittleness may result whenever material under very high stress is exposed to hydrogen. In high strength materials very high local stresses may be expected because of the complex microstructures associated with high strength levels. In lower strength materials plastic deformation may be expected to intervene before very high local stresses are developed. This suggests that whiskers of metals normally soft in bulk form might well be brittle in the presence of hydrogen gas, since very high stresses may be attained in whiskers. It also suggests that experimental studies of the interaction between hydrogen and unstressed material will not provide information directly relevant to hydrogen brittieness.

\section{ACKNOWLEDGEMENT}

It is a pleasure to aclinowledge the financial sup- port of the U. S. Atomic Energy Commission and the provision of facilities by the Materials Science Center, supported by the National Science Foundation. 


\section{REFERENCES}

(1) J. E. Campbe11: Effects of Hydrogen Gas on Metals at Ambient Temperature, DMIC Report S-31, Battelle Memorial Institute, Columbus, Ohio, 1970.

(2) G. G. Hancock and H. H. Johnson: Trans. Met. Soc. AIME, 1966, vol. 236, pp. 513-516.

(3) D. P. Williams and H. G. Nelson: Met. Trans., 1970, vol. 1, pp. 63-68.

(4) R. J. Walter, R. P. Jewett and W. T. Chandler: Mater. Sci. Eng. 1969/1970, vol. 5, pp. 98-110.

(5) R. J. Walter and W. T. Chandler: Mater. Sci. Eng., 1971, vol. 8, pp. 90-97.

(5) H. G. Nelson, D. P. Williams and J. E. Stein: Met. Trans., 1972, vo1. 3, pp. 469-475.

(7) D. P. Williams and H. G. Nelson: Met. Trans., 1972, vol. 3, pp. 2107-2113.

(8) A. W. Thompson and B. A. Wilcox: Scripta Metālurgica, vol. 6, pp. 689-696.

(9.) J. D. Frandsen, N. E. Paton and H. L. Marcus: Scripta Metallurgica, 1973, vol. 7, pp. 409-414.

(10) 0. D. Gonzalez, quoted by R. A. Oriani, Proceedings of Conference on Fundamental Aspects of Stress Corrosion Cracking, NACE, 1969, p. 43.

(11) H. H. Johnson, J. G. Morlet and A. R. Troiano: Trans. Met. Soc. AIME, 1958, vol. 212, pp. 526-541.

(12) R. A. Oriani: Met. Trans., 1970, vol. 1, p. 2346.

(13) V. Sawicki and H. Johnson: Met. Trans., 1971, vol. 2, p. 3496 .

(14) H. Hohnson: International Conference on Stress Corrosion Cracking and Hydrogen Embrittlement of Iron-Base Alloys, Firminy, France, 1973.

(15) H. H. Johnson: Proceedings of Conference on Fundamental Aspects of Stress Corrosion Cracking, National AsSociation of Corrosion Engineers, Houston, 1969, p. 439. 
(16) H. G. Nelson, D. P. Williams and A. S. Tetelman: Met. Trans., 1971, vol. 2, pp. 953-959.

(17) K. Farrell and A. G. Quarrell: J.I.S.I., 1964, vol. 202, pp. 1002-1011.

(18) J. B. Steinman, H. C. VanNess and.G. S. Anse11: Welding Journal, Welding Research Supplement, 1965, vol. $44, p p .221 s-224 s$.

(19) R. J. Walter and W: T. Chandler: paper presented at 1968 Western Metal and Tool Conference, Los Angeles, California, March 1968. ASM Report No. D8-74.2, ASM Report System, Metals Park, Ohio 44073.

(20) J. J.Gilman: Phil. Mag. (Ser. 8), 1972, vol. 26, pp. 801 .

(21) H. S. Carslaw and J. C. Jaeger: Conduction of Heat in Solids, 2nd ed., oxford University Press, London, T959, p. 267.

(22) R. W. Balluffi and D. N. Seidman: Jnl. Appl. Phys., 1965, vol. 36, pp. 2708-2720.

(23) C.Atkinson: Jn1.App 1. Phys., 1971, vol. 42, pp. 1994-1998.

(24) J. P. Fidelle: French Atomic Energy Commission, private communication.

(25) M. R. Louthan, Jr., G. R. Caskey, Jr., J.A. Donovan and D. E. Rawi, Jr.: Mater. SCi. Eng., 1972, vol. 10, pp. 357-368.

(26) J.C.M. Li, R. A. Oriani and L. S. Darken: Z. Phys. Chem. (N.F.), 1966, vol. 49, pp. 271-283.

(27) J. R. Rice and M. A. Johnson: Inelastic Behavior of Solids, McGraw-Hil1, 1970, pp.641-672. 


\section{FIGURE CAPTIONS}

Fig. 1. Concentration profile ahead of moving crack; moving line source model, crack tip radius $r_{0}=5 \times 10^{-8} \mathrm{~cm}$.

Fig. 2. Concentration profiles for crack modelled as moving line source and as moving semi-infinite source. 


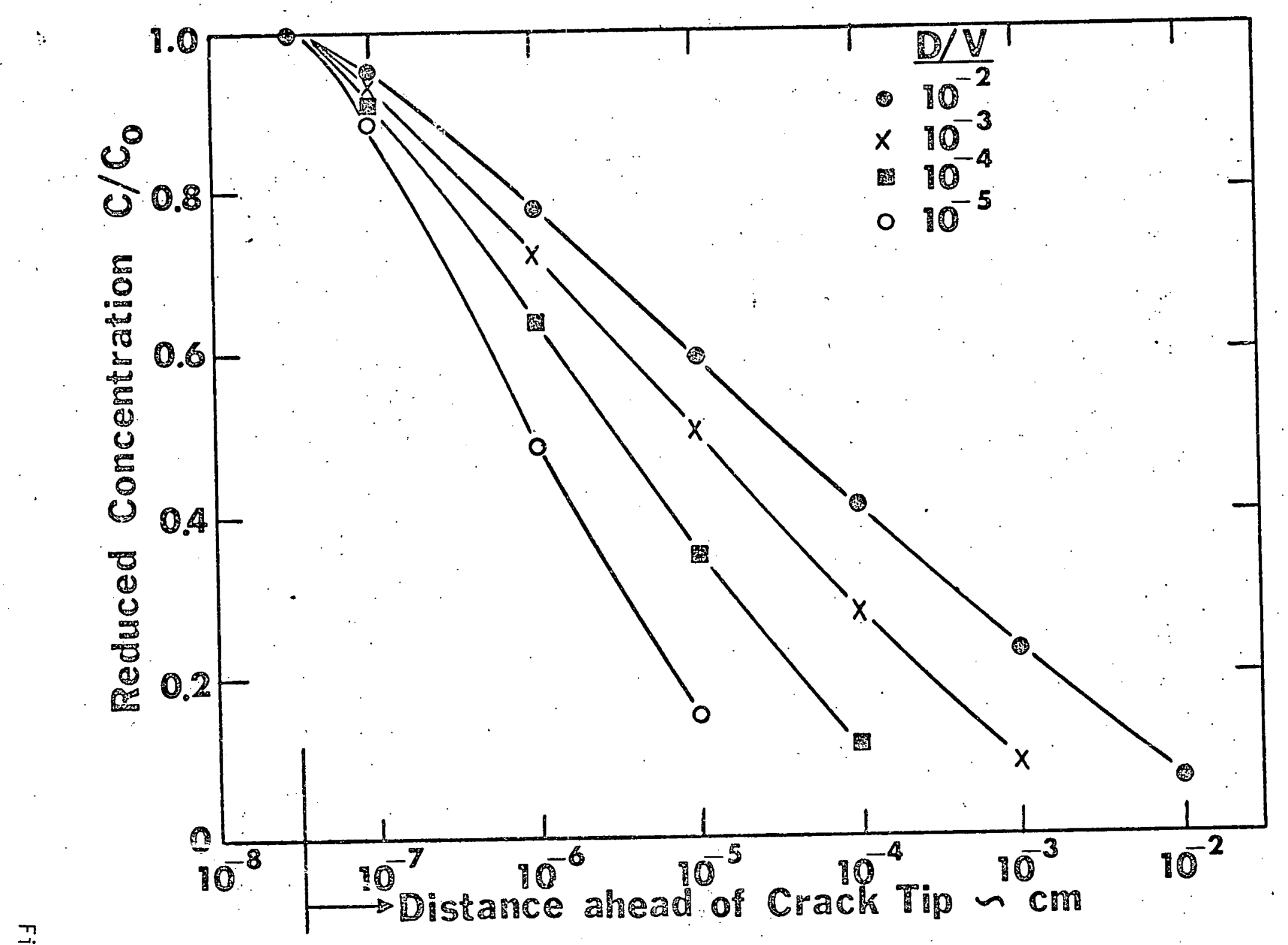

ㄱ. 


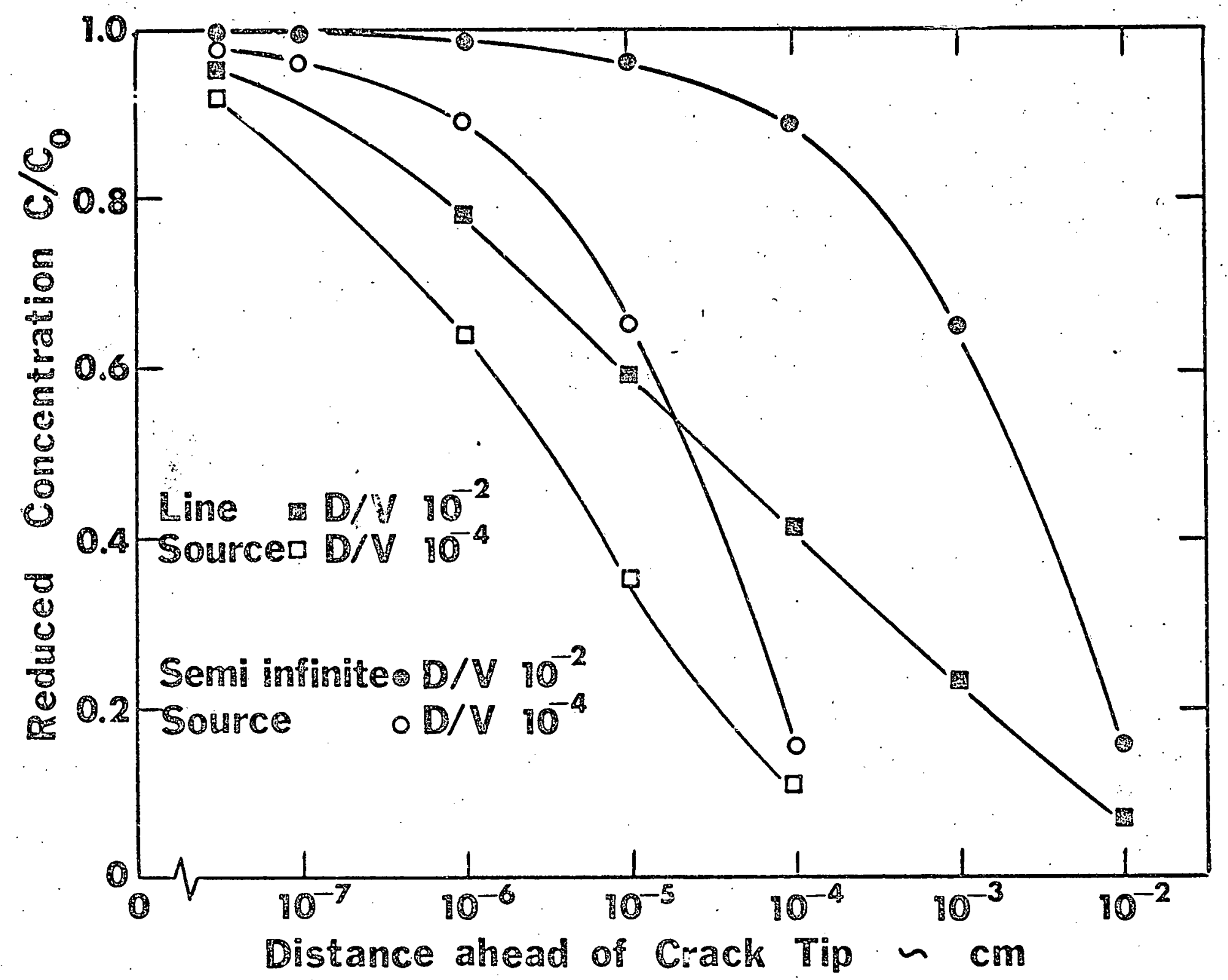

